Check for updates

Cite this: RSC Adv., 2018, 8, 805

Received 30th October 2017

Accepted 13th December 2017

DOI: 10.1039/c7ra11932a

rsc.li/rsc-advances

\title{
Changing molecular conjugation with a phenazine acceptor for improvement of small molecule-based organic electronic memory performance $\uparrow$
}

\author{
Quan Liu, (D) * Caibin Zhao, Guanghui Tian and Hongguang Ge
}

\begin{abstract}
Two conjugated small molecules with different molecular conjugation, 4', $4^{\prime \prime}$-(diazene-1,2-diyl)bis $\left(2^{\prime}, 3^{\prime}, 5^{\prime}, 6^{\prime}\right.$ tetrafluoro- $N, N$-diphenyl-[1,1'-biphenyl]-4-amine) (TPA-azo-TPA) and 4,4'-(perfluorophenazine-2,7-diyl) bis( $N, N$-diphenylaniline) (TPA-ph-TPA), in which the electron donor triphenylamine moiety is bridged using different electron-accepting azobenzene or phenazine blocks, were designed and synthesized. The TPA-phTPA molecule with a larger conjugation acceptor regularly formed a nanocrystalline film and the asfabricated memory devices exhibited outstanding non-volatile write once read many (WORM) memory effects with an ON/OFF ratio ten times higher than that of TPA-azo-TPA. Using theoretical calculations, it was speculated that the memory performance is a result of an electric field induced charge transfer effect and the enhanced device performance of the acceptor molecular conjugation is because of the presence of a strong charge transfer effect. The experimental findings suggest that the strategy of molecular conjugation may promote the performance of small molecule-based organic electronic memory devices by an enhanced a strong charge transfer effect.
\end{abstract}

\section{Introduction}

Traditional magnetic or optical material based data storage systems were limited by their memory capacity and cell size, which greatly restricted their use in applications for high density data storage. ${ }^{1-3}$ Organic electronic memory (OEM) is a promising alternative to achieve high-performance data storage and has gained significant scientific interest over the past few years. Many materials can be used for OEM, including polymeric materials, ${ }^{4,5}$ composite materials ${ }^{6-8}$ and small molecules. ${ }^{9-11}$ Among them, the organic small molecules have evoked considerable research interest because of their low cost, welldefined molecular structure, easy purification, and optoelectrical property tunability. ${ }^{\mathbf{1 2}}$

Recently, numerous high-performance organic semiconductors and various outstanding memory behaviors have been successfully achieved using innovative molecular designs. Yam et al. designed a series of subphthalocyanin-based and boron(III) diketonate-based molecules with tunable memory device performance. ${ }^{\mathbf{1 3 , 1 4}}$ In previous work, the strength of push-

Shaanxi Province Key Laboratory of Catalytic Foundation and Applications, School of Chemical and Environmental Science, Shaanxi University of Technology, Hanzhong, 723001, China. E-mail: liuq@snut.edu.cn; Tel: +869162641660

$\dagger$ Electronic supplementary information (ESI) available: MS and NMR spectra of compounds are listed in Fig. S2-S6. The $I-V$ characteristics of the memory devices using LiF as block layer are shown in Fig. S7. The long-term stability of the memory devices under ambient conditions are shown in Fig. S8. The memory behavior of the flexible memory devices under different bending conditions are shown in Fig. S9. See DOI: 10.1039/c7ra11932a pull electron groups was changed and this lead to the "charge trapping" hypothesis. Subsequently, tuning the length of the flexible alkyl side chain, molecular planarity, and conjugation length, was studied precisely to adjust the memory performances. ${ }^{15-17}$ Azobenzene (azo) because of its week electron withdrawing ability was commonly used for the "charge trapping" group in small molecule memory material. ${ }^{18-20}$ However, the stability and conjugate planarity of azobenzene are poor, which greatly limits their use because of their data storage performance. Phenazine (ph) derivatives play an important role in organic semiconductors for their wide range of photoelectric properties and stability. ${ }^{21-23}$ Therefore, it is important to design a new phenazine-based conjugate skeleton rather than one based on azobenzene.

In this work, first molecules linked with azobenzene (TPAazo-TPA) were synthesized, and then the azobenzene was replaced with phenazine (TPA-ph-TPA), as shown in Scheme 1. Both molecules have an electron-donating triphenylamine moiety, which is bridged by blocks with different conjugation abilities. The influences of molecular conjugation changed the film morphology, and the memory device performance were precisely and systematically investigated. The results showed that the sandwich memory device with the larger conjugation molecule (TPA-ph-TPA) as the active layer exhibited better binary electrical conductance switching and nonvolatile memory effects with long-term thermal stability than the smaller conjugation molecule (TPA-azo-TPA) (Scheme 2). 


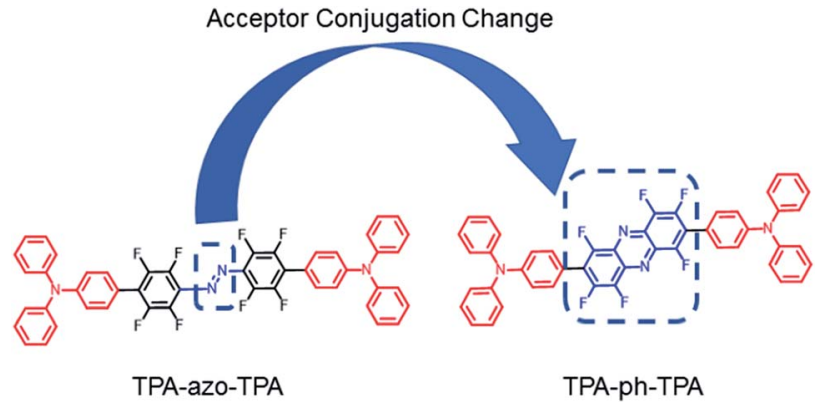

Scheme 1 Molecular structures of the TPA-azo-TPA and TPA-phTPA.

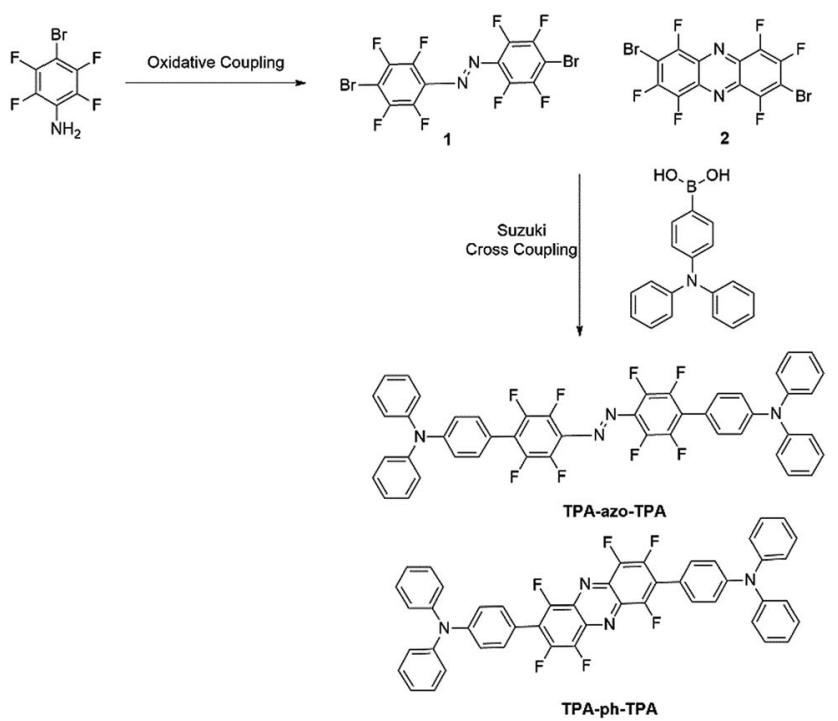

Scheme 2 Synthesis routes of TPA-azo-TPA and TPA-ph-TPA.

\section{Experimental}

\section{Materials}

4-Bromo-2,3,5,6-tetrafluoroaniline, hypochlorous acid tert-butyl ester $(t-\mathrm{BuOH}),[4-($ diphenylamino)phenyl]boronic acid, palladium diacetate, sodium iodide (NaI), potassium carbonate, $\left[1,1^{\prime}\right.$-bis(diphenylphosphino)ferrocene $]$ dichloropalladium(II) and potassium acetate were purchased from commercial sources (TCI, Alfa Aesar, and Sigma-Aldrich). All solvents were purchased from Sinopharm Chemical Reagent Co., Ltd. All chemicals were used as received without further purification.

\section{Characterization}

All electrical measurements of the devices were performed under ambient conditions without any encapsulation using an HP 4145B semiconductor parameter analyzer (Hewlett Packard) equipped with an HP 8110A pulse pattern generator (Hewlett Packard). Nuclear magnetic resonance (NMR) spectra were recorded using an Inova $400 \mathrm{MHz}$ Fourier transform (FT)-NMR spectrometer (Varian). High-resolution mass spectra were acquired using a Micromass GCT-time-of-flight (TOF) gas chromatography/mass (GC/MS) spectrometer (Waters) with an electrospray ionization source. The film thickness was determined using a Form Talysurf Intra Profiler (Taylor Hobson). Ultraviolet-visible (UV-vis) absorption spectra were measured at room temperature using a UV-3600 spectrophotometer (Shimadzu). Atomic force microscopy (AFM) measurements were performed using a MFP-3DTM AFM instrument (Digital Instruments/Asylum Research). Thermogravimetric analysis (TGA) was conducted using a Dynamic Rate method with a TGA 2950 (TA Instruments) at a heating rate of $10{ }^{\circ} \mathrm{C} \mathrm{min}^{-1}$ under

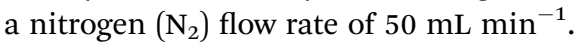

\section{Fabrication of memory devices}

The indium tin oxide (ITO) substrates were pre-cleaned sequentially with ethanol, acetone and isopropanol in an ultrasonic bath for $20 \mathrm{~min}$. The benzene solution of TPA-ph-TPA and TPA-azo-TPA was filtered through poly(tetrafluoroethylene) membrane micro-filters with a pore size of $0.32 \mu \mathrm{m}$. Then, $0.1 \mathrm{~mL}$ of solution was spin coated onto the substrates at a spinning speed of $500 \mathrm{rpm}$ for $10 \mathrm{~s}$ and then $2000 \mathrm{rpm}$ for $30 \mathrm{~s}$, followed by vacuum drying at $80{ }^{\circ} \mathrm{C}$ for $8 \mathrm{~h}$. The thickness of the films was about $90 \mathrm{~nm}$ as measured using a model M2000 DI spectroscopic ellipsometer (J. A. Woollam, USA). To construct the aluminum (Al)/TPA-ph-TPA and TPA-azo-TPA/ITO structures, Al top electrodes were thermally evaporated onto the film surface under $2 \times 10^{-6}$ Torr through a shadow mask with a thickness of about $60 \mathrm{~nm}$ and an area of $0.20 \mathrm{~mm}^{2}$.

Fabrication of flexible memory devices: using $30 \times 30 \mathrm{~mm}$ sized poly(ethylene terephthalate) (PET) as the flexible substrate, Al bottom electrodes were thermally evaporated onto the PET substrate under $2 \times 10^{-6}$ Torr through a shadow mask with a thickness of about $100 \mathrm{~nm}$ and an area of $1 \times 30 \mathrm{~mm}$. Then, an $80 \mathrm{~nm}$ thickness organic layer was vacuum deposited onto the bottom Al electrodes as measured using the spectroscopic ellipsometer. The Al top electrodes were thermally evaporated onto the organic layer through a shadow mask with a thickness of about $100 \mathrm{~nm}$ and an area of $1 \times 30 \mathrm{~mm}$.

\section{Synthetic procedures}

Synthesis of 1,2-bis(4-bromo-2,3,5,6-tetrafluorophenyl) diazene (1). Compound 1 was prepared according to a previous method found in the literature. ${ }^{24}$ To a mixture of 4 bromo-2,3,5,6-tetrafluoroaniline $(0.5 \mathrm{mmol})$ and NaI $(1.0 \mathrm{mmol}$, $150.0 \mathrm{mg})$ in $t$-BuOH $(3 \mathrm{~mL})$, was added tert-butyl hypochlorite $(1.0 \mathrm{mmol}, 108.6 \mathrm{mg})$ under an $\mathrm{N}_{2}$ atmosphere at room temperature for $3 \mathrm{~h}$. The mixture was stirred for the indicated time and quenched with aqueous sodium thiosulfate $(1.0 \mathrm{M}$, $10 \mathrm{~mL}$ ), and the solution was extracted with dichloromethane $\left(\right.$ DCM $\left.\left(\mathrm{CH}_{2} \mathrm{Cl}_{2}\right) ; 20 \mathrm{~mL} \times 3\right)$. The combined organic extracts were dried over sodium sulfate and concentrated under vacuum to give the crude product. Purification using flash column chromatography on silica gel [eluent: $\mathrm{CH}_{2} \mathrm{Cl}_{2}$ in petroleum ether (PE)] gave the product. ${ }^{19} \mathrm{~F}-\mathrm{NMR}[282 \mathrm{MHz}$, deuterated dimethylsulfoxide (DMSO- $\left.\left.d_{6}\right)\right] d(\mathrm{ppm}):-131.23(\mathrm{~d}, J=16.0 \mathrm{~Hz}$, $2 \mathrm{~F}),-133.64$ (d, $J=13.3 \mathrm{~Hz}, 2 \mathrm{~F}),-147.70(\mathrm{~d}, J=16.0 \mathrm{~Hz}, 2 \mathrm{~F})$, -148.61 (d, $J=13.3 \mathrm{~Hz}, 2 \mathrm{~F})$; MS: calc'd for $\mathrm{C}_{12} \mathrm{Br}_{2} \mathrm{~F}_{8} \mathrm{~N}_{2}[\mathrm{M}+\mathrm{H}]^{+}$ 
483.9370, found 483.8380. Anal. found for $\mathrm{C}_{12} \mathrm{Br}_{2} \mathrm{~F}_{8} \mathrm{~N}_{2}$ : C, 29.98; $\mathrm{Br}, 32.84 ; \mathrm{N}, 5.75$.

Synthesis of 2,7-dibromo-1,3,4,6,8,9-hexafluorophenazine (2). The synthesis method of 2 is similar to the method of synthesis for 1 .

${ }^{19}$ F-NMR (282 MHz, DMSO- $\left.d_{6}\right) d$ (ppm): $-118.49(\mathrm{~d}, J=$ $17.2 \mathrm{~Hz}, 2 \mathrm{~F}),-125.15$ (d, $J=18.3 \mathrm{~Hz}, 2 \mathrm{~F}),-151.82-151.95(\mathrm{~m}$, 2F). MS: calc'd for $\mathrm{C}_{12} \mathrm{Br}_{2} \mathrm{~F}_{6} \mathrm{~N}_{2}[\mathrm{M}+\mathrm{H}]^{+}$443.8332, found 443.8040. Anal. found for $\mathrm{C}_{12} \mathrm{Br}_{2} \mathrm{~F}_{6} \mathrm{~N}_{2}$ : C, 32.32; Br, 35.84; N, 6.27 .

General procedure for the synthesis of TPA-azo-TPA and TPA-ph-TPA. A solution of 1 or $2(0.409 \mathrm{~g}, 1 \mathrm{mmol}), 4$-(diphenylamino)phenylboronic acid $(0.723 \mathrm{~g}, 2.5 \mathrm{mmol})$ and tetrakis(triphenylphosphine)palladium $(0) \quad(0.062 \mathrm{~g}, \quad 0.05 \mathrm{mmol})$ dissolved in toluene $(15 \mathrm{~mL})$ was stirred in a two-necked flask under a $\mathrm{N}_{2}$ atmosphere for $30 \mathrm{~min}$. To the reaction mixture, potassium carbonate $(0.28 \mathrm{~g}, 2 \mathrm{mmol})$ in distilled water $(15 \mathrm{~mL})$ was added dropwise over a period of $20 \mathrm{~min}$. The resulting solution was refluxed overnight at $80{ }^{\circ} \mathrm{C}$. The reaction mixture was extracted with dichloromethane (DCM) and the organic layer was separated. The organic layer was evaporated with a rotary evaporator, and then the resulting powdery product was purified using column chromatography with DCM : PE (1:5) as the eluent and a crystalline solid was obtained.

\section{Spectral data}

$4^{\prime}, 4^{\prime \prime \prime}$-(Diazene-1,2-diyl)bis (2', 3', $5^{\prime}, 6^{\prime}$-tetrafluoro- $N, N$-diphenyl[1,1'-biphenyl]-4-amine) (TPA-azo-TPA). Yield 74\%, ${ }^{1} \mathrm{H}-\mathrm{NMR}[400$ $\mathrm{MHz}$, deuterated chloroform $\left.\left(\mathrm{CDCl}_{3}\right)\right] d(\mathrm{ppm}): 7.41-7.31(\mathrm{~m}$, 12H), 7.20-7.05 (m, 16H); ${ }^{13} \mathrm{C}-\mathrm{NMR}\left(100 \mathrm{MHz}, \mathrm{CDCl}_{3}\right) d$ (ppm): $147.3,146.9$, 132.0, 131.0, 129.5, 129.4, 129.3, 125.5, 125.4, 124.9, 124.1, 123.4, 122.1, 121.3; ${ }^{19} \mathrm{~F}-\mathrm{NMR}$ (376 MHz, $\mathrm{CDCl}_{3}$ ) $d$ (ppm): 111.6, 127.1, 144.2, 149.6; TOF-MS: calc'd for $\mathrm{C}_{48} \mathrm{H}_{28} \mathrm{~F}_{8} \mathrm{~N}_{4}[\mathrm{M}+\mathrm{H}]^{+}$812.2186, found 812.2000; anal. found for $\mathrm{C}_{48} \mathrm{H}_{28} \mathrm{~F}_{8} \mathrm{~N}_{4}$ : C, 70.65; H, 3.49; N, 6.92.

4,4'-(perfluorophenazine-2,7-diyl)bis( $N, N$-diphenylaniline) (TPAph-TPA). Yield: $82 \%,{ }^{1} \mathrm{H}-\mathrm{NMR}\left(400 \mathrm{MHz}, \mathrm{CDCl}_{3}\right) d(\mathrm{ppm}): 7.70(\mathrm{~s}$, 2H), 7.54-7.52 (m, 4H), 7.34-7.33 (m, 6H), 7.24-7.18 (m, 12H), 7.15-7.13 (m, 4H); ${ }^{13} \mathrm{C}-\mathrm{NMR}\left(100 \mathrm{MHz}, \mathrm{CDCl}_{3}\right) d(\mathrm{ppm}): 146.4$, 144.4, 131.0, 129.1, 128.9, 125.2, 123.7, 120.8, 104.5; ${ }^{19} \mathrm{~F}-\mathrm{NMR}$ (376 $\left.\mathrm{MHz}, \mathrm{CDCl}_{3}\right) d$ (ppm): 129.2, 130.9, 153.1; TOF-MS: calc'd for $\mathrm{C}_{48} \mathrm{H}_{28} \mathrm{~F}_{6} \mathrm{~N}_{4}[\mathrm{M}+\mathrm{H}]^{+} 774.2218$, found 774.2210; anal. found for $\mathrm{C}_{48} \mathrm{H}_{28} \mathrm{~F}_{6} \mathrm{~N}_{4}$ : C, 74.70; H, 3.66; N, 7.26.

\section{Results and discussion}

\section{Thermal stability}

The molecules TPA-ph-TPA and TPA-azo-TPA can be obtained in moderate yields using a multistep Suzuki cross-coupling reaction. The thermal decomposition temperature of the TPA-azoTPA is $261{ }^{\circ} \mathrm{C}$, however, TPA-ph-TPA exhibits good thermal stability with a decomposition temperature above $300{ }^{\circ} \mathrm{C}$ (Fig. 1a). Compared with the currently reported azo-based small molecules, ${ }^{25-27}$ the stability of TPA-ph-TPA was good. This suggested that increasing the degree of conjugation of the molecular framework leads to the further improvement of the molecular heat resistance.
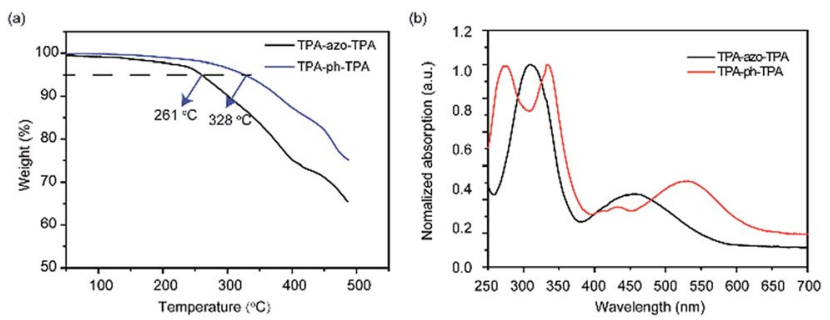

Fig. 1 (a) TGA curves of TPA-azo-TPA, and TPA-ph-TPA at a heating rate of $10{ }^{\circ} \mathrm{C}$ min $^{-1}$ under a $\mathrm{N}_{2}$ atmosphere; (b) UV-vis absorption spectrum of TPA-azo-TPA, and TPA-ph-TPA in DCM solution.

\section{Photophysical and electrochemical properties}

The TPA-ph-TPA and TPA-azo-TPA have good solubility and so DCM was chosen as the solvent and a spin coating method was used to fabricate the film. Fig. 1b shows the optical absorption spectra of TPA-ph-TPA and TPA-azo-TPA nano-films on quartz substrates. The strong absorption bands at approximately 400$500 \mathrm{~nm}$ can be attributed to the $\mathrm{n}-\pi^{*}$ transition (charge transfer) of the azobenzene. ${ }^{28}$ Compared with that of TPA-azo-TPA, the onset optical absorbance of TPA-ph-TPA exhibits a significant red-shift for $34 \mathrm{~nm}$, which corresponds to the narrower energy band gap of the phenazine structure. The optical band gaps of the TPA-ph-TPA and TPA-azo-TPA molecules, estimated from the absorption edges of the films, were $2.03 \mathrm{eV}$ and $2.15 \mathrm{eV}$, respectively. Therefore, increased conjugation with the phenothiazine structure gives rise to a ground state charge transfer complex in TPA-ph-TPA. This also suggests that the TPA-ph-TPA film forms an ordered stacking of the $\pi$-conjugation system, favouring an effective carrier migration.

Fig. 2 shows the cyclic voltammetry (CV) measurements of TPAazo-TPA and TPA-ph-TPA on an ITO glass substrate in a $0.1 \mathrm{~mol} \mathrm{~L}^{-1}$ solution of tetrabutylammonium hexafluorophosphate $\left(\mathrm{TBAPF}_{6}\right)$ in anhydrous acetonitrile $\left(\mathrm{CH}_{3} \mathrm{CN}\right)$ solution and measurements were taken with a scan rate of $100 \mathrm{mV} \mathrm{s}^{-1}$. The onset oxidation ( $E_{\mathrm{ox}}^{\text {onset }}$ ) of TPA-azo-TPA is approximately $0.85 \mathrm{~V}$ versus silver/silver chloride $(\mathrm{Ag} / \mathrm{AgCl})$. The onset oxidations ( $E_{\mathrm{ox}}^{\text {onset }}$ ) of TPA-ph-TPA were $0.35 \mathrm{~V}$ and $0.83 \mathrm{~V}$ versus $\mathrm{Ag} / \mathrm{AgCl}$, respectively. The oxidation potential onset of ferrocene $E_{\mathrm{Fe}}^{\text {onset }}$ was $0.44 \mathrm{~V}$ versus $\mathrm{Ag} / \mathrm{AgCl}$ in acetonitrile with bare ITO glass. The estimated highest occupied molecular orbital (HOMO) levels can be calculated from the $E_{\mathrm{Ox}}^{\text {onset }}$ using the following formula: $\mathrm{HOMO}=-\left[E_{\mathrm{Ox}}^{\text {onset }}+4.8-E_{\mathrm{Fc}}\right] \mathrm{eV}$. The lowest unoccupied molecular orbital (LUMO) levels are not detectable using CV and so the values of the LUMO levels were estimated using the following formula: LUMO $=\left[\mathrm{HOMO}+E_{\mathrm{g}}\right] \mathrm{eV}$. The HOMO levels of TPA-azo-TPA and TPA-ph-TPA were $-5.21 \mathrm{eV}$ and $-4.72 \mathrm{eV}$, respectively. The determined LUMO levels of TPAazo-TPA and TPA-ph-TPA were $-3.06 \mathrm{eV}$ and $-2.69 \mathrm{eV}$, respectively. TPA-ph-TPA has lower LUMO levels than TPA-azo-TPA, because of the charge transfer process, and this result was in agreement with the UV spectroscopic data.

\section{Morphology of the thin film}

To investigate the surface morphology and film microstructure, AFM measurements were made on the TPA-azo-TPA and TPA- 


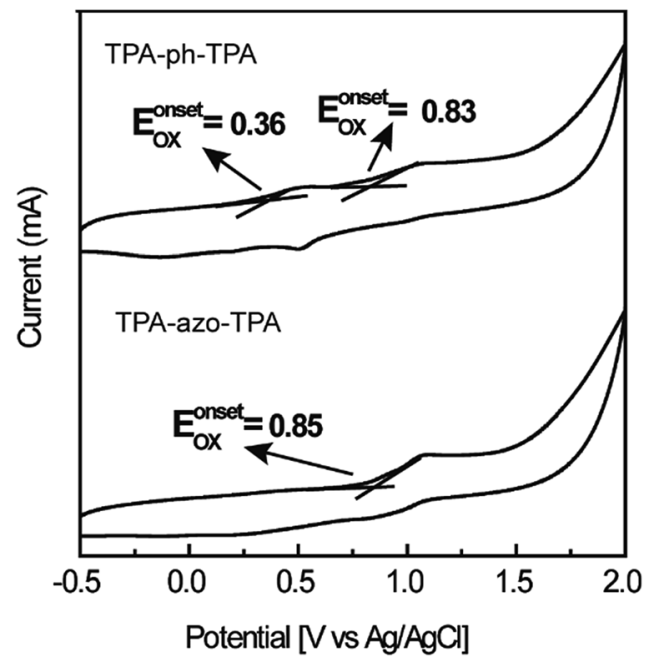

Fig. 2 Cyclic voltammograms of TPA-azo-TPA and TPA-ph-TPA film were measured in $0.1 \mathrm{mmol} \mathrm{L}^{-1} \mathrm{TBAPF}_{6} / \mathrm{CH}_{3} \mathrm{CN}$ solution with $\mathrm{Ag} / \mathrm{AgCl}$ as reference electrode and platinum wire as the counter electrode. $\mathrm{A}$ scan rate of $100 \mathrm{mV} \mathrm{s}^{-1}$ was used.

ph-TPA film. As seen from the AFM height images (Fig. 3), both of the films showed a relatively smooth surface without obvious defects. The TPA-azo-TPA film clearly showed two-dimensional grains in its solid state at room temperature with uniform size and the surface root-mean-square (RMS) roughness was $1.8 \mathrm{~nm}$. The TPA-ph-TPA film showed a denser needle morphology, and had a RMS roughness of $0.9 \mathrm{~nm}$, which was smaller than that of TPA-azo-TPA. Therefore, the film quality was improved significantly.

\section{Current-voltage $(I-V)$ characteristics of the memory devices}

The memory effect of the TPA-azo-TPA devices was explored first, and the current-voltage $(I-V)$ characteristics are shown in Fig. 4a. Under a negatively biased voltage sweep, the current in the fabricated Al/TPA-azo-TPA/ITO memory devices increased abruptly from $10^{-5} \mathrm{~A}$ to $10^{-2} \mathrm{~A}$ at around $3.0 \mathrm{~V}$, indicating that the device has been switched from an OFF state to an ON state (sweep 1 of Fig. 4a). This transition can be defined as the "Write" process and an ON/OFF ratio of $10^{3}$ can be obtained. The device remained in the ON state, even after the power was turned off or during the subsequent voltage sweep (sweep 2).

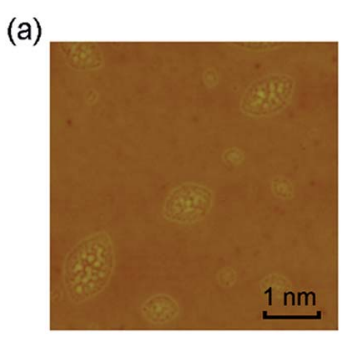

(b)

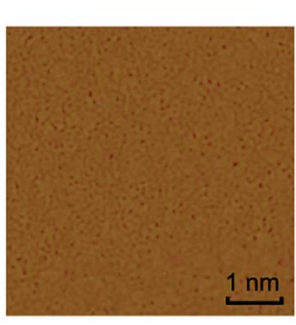

$50 \mathrm{~nm}$

Fig. 3 Tapping mode $(5 \mu \mathrm{m} \times 5 \mu \mathrm{m})$ AFM phase of TPA-azo-TPA (a) and TPA-ph-TPA (b) film on ITO substrates.
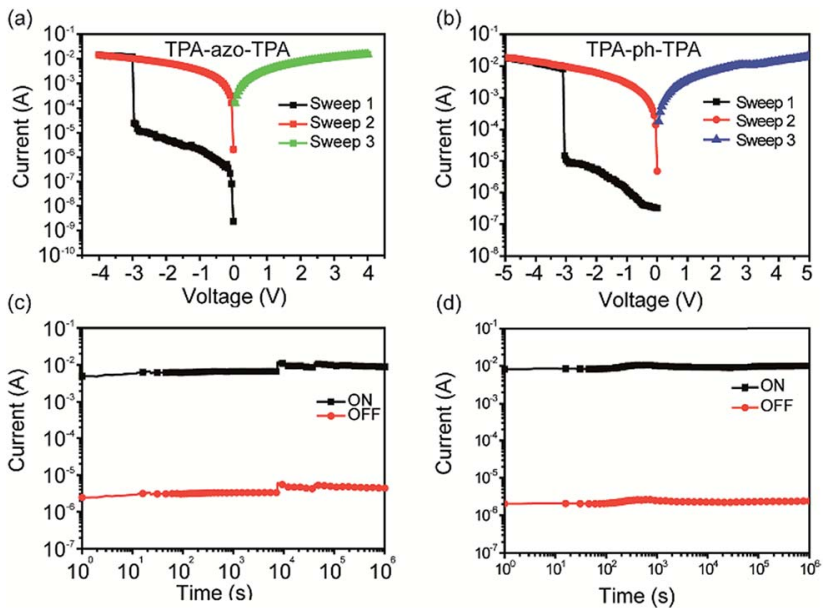

Fig. $4 \quad I-V$ characteristics of the small molecule-based memory devices: (a) Al/TPA-azo-TPA/ITO and (b) Al/TPA-ph-TPA/ITO; the effect of retention time of (c) the Al/TPA-azo-TPA/ITO and (d) the Al/ TPA-ph-TPA/ITO memory devices under a constant stress of $-1.0 \mathrm{~V}$.

The ON state was maintained in the final sweep from 0 to $-4 \mathrm{~V}$ (sweep 3). Therefore, the TPA-azo-TPA-based device exhibited a typical nonvolatile write once read many (WORM) memory behavior. Compared with the Al/TPA-azo-TPA/ITO devices, the Al/TPA-ph-TPA/ITO devices exhibited much smoother $I-V$ characteristics during the switching process. As shown in Fig. $4 \mathrm{~b}$, the Al/TPA-ph-TPA/ITO memory cell was in the OFF state. When a negatively biased potential sweep from 0 to $-4 \mathrm{~V}$ was applied (sweep 1 in Fig. 4b), the current increased consecutively from $10^{-6} \mathrm{~A}$ to $10^{-2} \mathrm{~A}$, indicating that the device had been set to the ON state by the negative forward sweep. The device remained in the ON state during the subsequent voltage sweep (sweep 2). In the following positive sweep from 0 to $4 \mathrm{~V}$ (sweep 3), the device remained in the ON state. The value of the ON/OFF ratio for TPA-ph-TPA-based devices was $10^{4}$. The TPAazo-TPA-based device also showed a typical nonvolatile WORM memory performance. Both of these two devices have potential applications for non-volatile data storage. Compared with the Al/TPA-azo-TPA/ITO device, the TPA-ph-TPA-based device has a higher ON/OFF ratio, which is helpful for avoiding false programming and error readout problems.

In order to exclude the possibility of the influence of metal filaments in the memory behaviours, sandwich structured memory devices: Al/LiF/small molecules/ITO were fabricated using the same conditions. The LiF layer $(5 \mathrm{~nm})$ acted as a buffer layer to prevent the $\mathrm{Al}$ nanoparticles penetrating into the active films. The $I-V$ results indicated the WORM memory behaviours of TPA-azo-TPA and TPA-ph-TPA based memory devices, as shown in Fig. S1 and S2 (ESI $\dagger$ ), which demonstrated that the WORM memory behaviours were not dependent on the metal filament.

The retention times of the ON state and the OFF state of the Al/TPA-azo-TPA/ITO and Al/TPA-ph-TPA/ITO device is shown in Fig. $4 \mathrm{c}$ and $\mathrm{d}$. Under a constant stress of $-1 \mathrm{~V}$, TPA-ph-TPAbased devices were observed to have more stability and no significant degradation in current for any of the states at least 
$10^{6} \mathrm{~s}$ during the readout test. The long-term stability of two molecular-based devices under air and humidity conditions was measured, and the results of this are shown in Fig. S3 and S4 (ESI $\dagger$ ). The test results show that the TPA-ph-TPA-based devices have better long-term stability than the TPA-ph-TPA-based devices. This may be because the phenazine group is more stable than the azobenzene group in air and humidity conditions.

Because of the rigid planarity of the glass substrates and the brittleness of the ITO electrodes, which limits its application on flexible devices. Flexible substrates such as PET were used to replace the ITO glasses and to fabricate flexible memory device. ${ }^{29-31}$ The specific fabricated method is described in the Experimental section. The flexible memory device can be bent under different conditions and the degree of bending was defined by the distance $(D)$ between the ends of the arc. The top and bottom $\mathrm{Al}$ electrodes of this flexible device were aligned perpendicularly to each other, and the voltage bias was applied to the memory devices at the end of the top $\mathrm{Al}$ electrode (cathode).

To confirm the feasibility of flexible memory devices, the sandwich structured flexible memory device with a 10 (word line) $\times 10$ (bit line) crossbar array is shown in Fig. 5a. The electric properties were measured under different bending conditions. For the TPA-azo-TPA-based flexible memory device (Fig. S5; ESI $\dagger$ ), the storage units of different regions could show stable binary memory behavior even when the distance between the ends of the arc bending was $20 \mathrm{~mm}$. When $D$ reached $10 \mathrm{~mm}$, the storage units of the TPA-azo-TPAbased flexible memory device became unstable. Additionally, the $I-V$ curves of the Al/TPA-ph-TPA/Al/PET structured memory device were also measured to test its flexibility. As shown in Fig. 5b, the device also maintained stable binary memory characteristics when $D$ was $10 \mathrm{~mm}$. The electrical characteristics of each curve are summarized from results obtained from 20 different cells under each bending condition.

The $I-V$ characteristics of flexible memory device over bending cycles of 500 times were also measured. The degree of bending was from the flat state to the maximum degree of bending $(D=10 \mathrm{~mm})$ and as the number of bending cycles increased, the TPA-azo-TPA-based flexible memory device cannot maintain stable binary memory behaviour, as shown in Fig. S6 (ESI $\dagger$ ). However, the TPA-ph-TPA-based flexible memory device showed stable binary memory behaviour up to 500 bending cycles of, as shown in Fig. 5c. In addition, the threshold voltage is a little larger than the previous bending and therefore shows a little shifting after several cycles of bending. This is probably because of an increase in the number of bending times, and the film cannot withstand the mechanical stretch and the ordered molecular stacking might be somehow damaged. Thus, the charges may have difficulty in moving through the films and much energy is needed to overcome the barrier. For bending cycles above 500, the TPAph-TPA-based memory device showed no visible binary memory behaviour. (a)

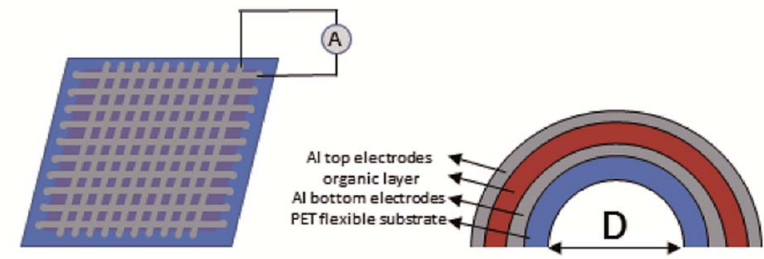

(b)

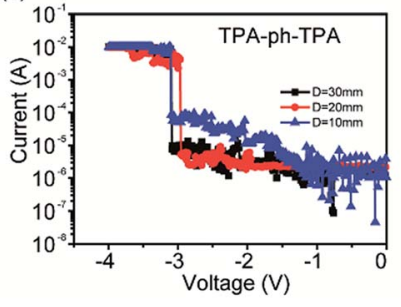

(c)

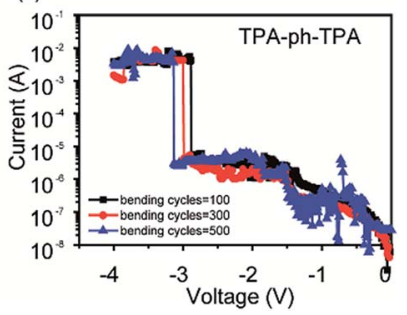

Fig. 5 (a) The model of the sandwich structure flexible memory device consisting of an active layer between the top and bottom Al electrodes (left), the calculation of the bending degree of the flexible memory device (right); (b) the memory effects of the TPA-ph-TPA-based flexible memory devices under different degrees of bending and the memory device can maintain the memory effect when the distance between the ends of the arc reaches $10 \mathrm{~mm}$. (c) The TPA-ph-TPAbased device can maintain memory performance within 500 bending cycles in the maximum bending condition.

\section{Memory mechanism}

To better understand the electronic process occurring inside the thin film, theoretical calculations were performed using density-functional program $\mathrm{DMol}_{3}{ }^{32,33}$ The hybrid functional B3LYP ${ }^{34,35}$ together with a double numerical plus polarization (DNP) basis set, was used. Comparing the $E_{\text {gap }}$ of TPA-azo-TPA and TPA-ph-TPA, the TPA-ph-TPA has a lower $E_{\text {gap }}$, which makes charge carrier migration easier. The molecular orbitals (HOMO, LUMO) and the electrostatic surface potential (ESP) of TPA-azoTPA and TPA-ph-TPA molecules were plotted, and are shown in Fig. 6.

To better understand the charge carrier behavior of TPA-azoTPA and TPA-ph-TPA based devices, the work functions (F) of Al top and ITO bottom electrodes were compared with molecular HOMO and LUMO orbital. The energy barrier between $F$ of the ITO $(-4.8 \mathrm{eV})$ and the HOMO energy level was $0.35-0.41 \mathrm{eV}$, which is much lower than the energy barrier of 1.21-1.39 eV between the $F$ of the $\mathrm{Al}(-4.3 \mathrm{eV})$ and the LUMO energy level. This suggests that hole injection from ITO into the HOMO of TPA-azo-TPA or TPA-ph-TPA (corresponding to ITO as the anode) is easier than electron injection from $\mathrm{Al}$ into the LUMO of TPA-azo-TPA or TPA-ph-TPA. Thus, TPA-azo-TPA and TPA-phTPA are p-type materials and holes predominate the conduction process (as shown in the inset of Fig. 6).

The ESP plots of TPA-azo-TPA and TPA-ph-TPA molecules both show an open channel along the molecular backbone with a continuous positive electrostatic potential, providing a path to allow charge carrier migration. For the TPA-azo-TPA molecule, electrons transit readily from the HOMO orbital to the LUMO orbital under an external electric field, forming the locally excited state. As a result, a charge-transfer interaction can occur in the TPA-azo-TPA molecule between the electron donor 
(a)

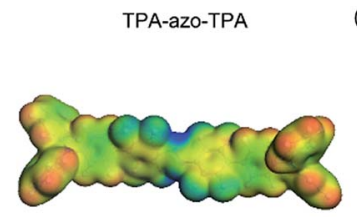

(b)

(c)
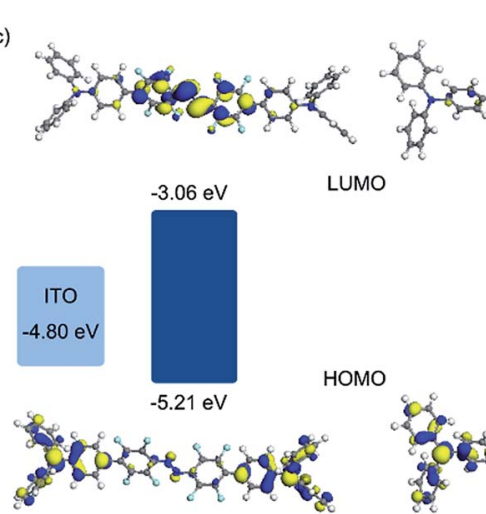

uмо

(8)

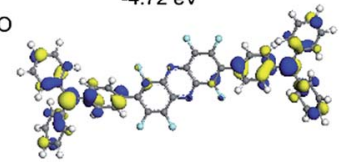

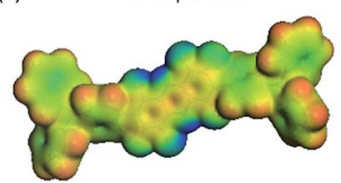

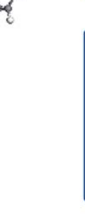

Fig. 6 Simulated electrostatic potential (ESP) plots of TPA-azo-TPA (a) and TPA-ph-TPA (b); HOMO, LUMO and energy level diagrams for the ITO/TPA-azo-TPA and TPA-ph-TPA/Al devices (c).

moieties and the electron acceptor moieties. For the TPA-phTPA molecule, a similar charge transfer process is also formed. However, the phenazine conjugation structure enhances the electron-withdrawing ability of the acceptor moiety, so the HOMO and LUMO orbitals of TPA-ph-TPA molecules show more obvious separation in the ground state, with HOMO mostly localizing on the donor areas and LUMO mostly localizing on the acceptor areas. It was speculated that the mechanism of the memory performance of the TPA-azo-TPA and TPA-ph-TPA devices could be the electric field induced charge transfer effect. Without an external electric field, the electrons in the TPA-azoTPA and TPA-ph-TPA molecules were stable, and the asfabricated device was in the OFF state. When a negative bias is applied, the charge transport pathways will form, and will switch the Al/TPA-azo-TPA or TPA-ph-TPA/ITO device from the OFF state to the ON state. However, the phenazine conjugation structure can significantly enhance the electron-withdrawing ability of the molecule, resulting in a stronger intramolecular charge transport in TPA-ph-TPA. It has been reported that changing the charge transfer ability of the D-A molecules can tune memory effects. So the better performance (larger ON/OFF ratio and more stability with each electric conductive state) of the TPA-ph-TPA-based device could be because of the stronger intramolecular charge transfer effect. The nonvolatile nature of the ON state is because of the intensive electron delocalization in the acceptor moieties which stabilized the conductive charge transfer state.

\section{Conclusions}

In conclusion, new materials based on fluorine substituted azobenzene- $\pi$-triphenylamine derivatives (TPA-azo-TPA) and fluorine substituted phenazine- $\pi$-triphenylamine derivatives
(TPA-ph-TPA) were synthesized. The photophysical, electrochemical properties and memory behaviors of these donor- $\pi$ acceptor molecules were investigated and compared. This comparative study of tuning the properties of the conjugated D-A-D molecules using an aromatic acceptor may be an alternative approach for the design and study of future highperformance memory devices based on new materials.

\section{Conflicts of interest}

There are no conflicts to declare.

\section{Acknowledgements}

The authors thank the Chinese Natural Science Foundation (21503125); the Scientific Research Program of the Shaanxi Provincial Education Department (2013JK0658 and No. 15JF013); the Brainstorm Project on Social Development by the Department of Science and Technology of Shaanxi Province (2015SF270) and the Doctoral Program of Shaanxi University of Technology (SLGQD17-15).

\section{References}

1 B. D. R. Chau, S. Datta, J. Kavalieros and K. Zhang, Nat. Mater., 2007, 6, 810-812.

2 E. Vogel, Nat. Nanotechnol., 2007, 2, 25-32.

3 E. Leobandung, L. Guo and S. Y. Chou, Science, 1997, 275, 649-651.

4 Y. Cheng, Y. Hou, T. Hobson and J. Liu, Nano Lett., 2010, 10, 2727-2733.

5 B. Hu, C. Wang and Q. Zhang, Chem. Sci., 2014, 5, 3404-3408.

6 S. Lee, Y. Jung, A. T. Jennings and R. Agarwal, Nano Lett., 2008, 8, 2056-2062.

7 P. Gu, C. Wang, B. Hua and Q. Zhang, J. Mater. Chem. C, 2015, 3, 10055-10065.

8 J. Xiao, Z. Yin, Y. Wu, J. Guo, Y. Cheng, H. Zhang and Q. Zhang, Small, 2011, 7, 1242-1246.

9 C. Wang, J. Wang, P. Li, J. Gao, S. Y. Tan, W. W. Xiong, B. Hu, P. S. Lee, Y. Zhao and Q. Zhan, Chem.-Asian J., 2014, 9, 779783.

10 C. Wang, P. Gu, B. Hu and Q. Zhang, J. Mater. Chem. C, 2015, 3, 10055-10065.

11 C. Wang, B. Hu, J. Wang and Q. Zhang, Chem.-Asian J., 2015, 10, 116-119.

12 D. M. Delongchamp, R. J. Kline, D. A. Fischer, L. J. Richter and M. F. Toney, Adv. Mater., 2011, 23, 319-337.

13 C. T. Poon, D. Wu, W. H. Lam and V. W. Yam, Angew. Chem., Int. Ed., 2015, 54, 10569-10573.

14 H. Chan, H. L. Wong, M. Ng, C. T. Poon and V. W. Yam, J. Am. Chem. Soc., 2017, 139, 7256-7263.

15 Q. Zhang, H. Zhuang, Y. Zhu, X. Xu, H. Liu, N. Li, Q. Xu, H. Li, J. Lu and L. Wang, J. Mater. Chem. C, 2013, 1, 38163824.

16 Q. Zhou, H. Zhuang, Y. Li, Q. Zhang, H. Li, Q. Xu, N. Li, J. Lu and L. Wang, ACS Appl. Mater. Interfaces, 2014, 6, 94-100. 
17 D. Wang, X. Wang, G. Zhou, W. Yu, Y. Zhou, Q. Fang and M. Jiang, J. Mater. Chem., 2001, 11, 1600-1605.

18 S. Miao, H. Li, Q. Xu, Y. Li, S. Ji, N. Li, L. Wang, J. Zheng and J. Lu, Adv. Mater., 2012, 24, 6210-6215.

19 S. Miao, H. Li, Q. Xu, N. Li, J. Zheng, R. Sun, J. Lu and C. M. Li, J. Mater. Chem., 2012, 22, 16582.

20 Y. Zhu, S. Miao, H. Zhuang, X. Xu, H. Li, R. Sun, N. Li, S. Ji and J. Lu, J. Mater. Chem. C, 2013, 1, 2320-2327.

21 P. Y. Gu, F. Zhou, J. Gao, G. Li, C. Wang, Q. F. Xu, Q. Zhang and J. M. Lu, J. Am. Chem. Soc., 2013, 135, 14086-14089.

22 P. Y. Gu, Y. Ma, J. H. He, G. Long, C. Wang, W. Chen, Y. Liu, Q. F. Xu, J. M. Lu and Q. Zhang, J. Mater. Chem. C, 2015, 3, 3167-3172.

23 J. Gao, P. Y. Gu, C. J. Lu, W. Chen, C. Wang, G. Li, F. Zhou, Q. F. Xu, J. M. Lu and Q. Zhang, Mater. Horiz., 2014, 1, 446451.

24 S. Okumura, C. H. Lin, Y. Takeda and S. Minakata, J. Org. Chem., 2013, 78, 12090-12105.

25 Y. Zhang, H. Zhuang, Y. Yang, X. Xu, Q. Bao, N. Li, H. Li, Q. Xu, J. Lu and L. Wang, J. Phys. Chem. C, 2012, 116, 22832-22839.
26 W. Ren, Y. Zhu, J. Ge, X. Xu, R. Sun, N. Li, H. Li, Q. Xu, J. Zheng and J. Lu, Phys. Chem. Chem. Phys., 2013, 15, 9212-9218.

27 Q. Liu, H. Dong, Y. Li, H. Li, D. Chen, L. Wang, Q. Xu and J. Lu, Chem.-Asian J., 2016, 11, 512-519.

28 Y. Ma, X. Cao, G. Li, Y. Wen, Y. Yang, J. Wang, S. Du, L. Yang, H. Gao and Y. Song, Adv. Funct. Mater., 2010, 20, 803-810.

29 Y. Yang, J. Ouyang, L. Ma, R. J.-H. Tseng and C.-W. Chu, Adv. Funct. Mater., 2006, 16, 1001-1014.

30 J.-M. Lu, Q.-J. Zhang, J.-H. He, H. Zhuang, H. Li, N.-J. Li, Q.-F. Xu and D.-Y. Chen, Chem.-Asian J., 2016, 11, 16241630.

31 A.-D. Yu, T. Kurosawa, Y.-H. Chou, K. Aoyagi, Y. Shoji, T. Higashihara, M. Ueda, C.-L. Liu and W.-C. Chen, ACS Appl. Mater. Interfaces, 2013, 5, 4921-4929.

32 B. Delley, J. Chem. Phys., 1990, 92, 508-517.

33 B. Delley, J. Chem. Phys., 2000, 113, 7756-7764.

34 D. Becke, J. Chem. Phys., 1993, 98, 5648-5652.

35 P. Stephens, F. Devlin, C. Chabalowski and M. J. Frisch, J. Phys. Chem., 1994, 98, 11623-11627. 